\title{
Polyubiquitin Promoter-Based Binary Vectors for Overexpression and Gene Silencing in Lotus japonicus
}

\author{
Takaki Maekawa, ${ }^{1,2}$ Mitsumasa Kusakabe, ${ }^{2}$ Yoshikazu Shimoda, ${ }^{3}$ Shusei Sato, ${ }^{3}$ Satoshi Tabata, ${ }^{3}$ \\ Yoshikatsu Murooka, ${ }^{2}$ and Makoto Hayashi ${ }^{1,2}$ \\ ${ }^{1}$ Institut für Genetik, Ludwig-Maximilians-Universität München, Maria-Ward-Str. 1a, 80638 München, Germany; ${ }^{2}$ Department \\ of Biotechnology, Graduate school of Engineering, Osaka University, Yamadaoka 2-1, Suita Osaka 565-0871, Japan; \\ ${ }^{3}$ Kazusa DNA Research Institute, 2-6-7 Kazusa-kamatari, Kisarazu, Chiba 292-0818, Japan
}

Submitted 16 May 2007. Accepted 4 December 2007.

In this study, we compared the transcriptional activities between Cauliflower mosaic virus (CaMV)35S promoter and polyubiquitin (Ljubq1) promoter from Lotus japonicus using $\beta$-glucuronidase (gus) reporter gene in transgenic plants of $L$. japonicus. The promoter analysis demonstrated that the Ljubq1 promoter possessed higher activity than the CaMV35S promoter in leaves, stems, roots, nodules, and pollen. Finally, we created GATEWAY conversion technology-compatible binary vectors for over-expression and RNA interference under the Ljubq1 promoter. These materials could provide alternative choice for studies in L. japonicus.

Strong promoters have been used for various applications in molecular biology, such as overexpression and RNA interference (RNAi). In addition to the high transcriptional activity, ubiquitous expression in most cell types often is demanded for these applications. In plants, the $35 \mathrm{~S}$ promoter from Cauliflower mosaic virus (CaMV35S promoter) fulfills these requirements in several species. However, in Lotus japonicus, although used for the molecular studies of root nodule symbiosis (Handberg and Stougaard 1992; Udvardi et al. 2005), the activity of the CaMV35S promoter in nodules generally is shown to be weak in other species (Auriac and Timmers 2007; Obertello et al. 2005; Quandt et al. 1993; Samac et al. 2004).

As an alternative to CaMV35S promoter, the high transcriptional activity of polyubiquitin promoters has been investigated in plants. Ubiquitin is involved in protein degradation in conjunction with the $26 \mathrm{~S}$ proteasome, which recognizes ubiquitin-conjugated proteins (Smalle and Vierstra 2004). The polyubiquitin gene encodes a tandem repeat of ubiquitin and is expressed in various tissue types of plant (Binet et al. 1991; Burke et al. 1988; Kawalleck et al. 1993; Wang et al. 2000). The genomic structure of polyubiquitin is conserved among various plants, including Helianthus annuus (sunflower), Zea mays (maize), Petroselinum crispum (parsley), Arabidopsis thaliana, Nicotiana tabacum (tobacco), Solanum tuberosum (potato), Pisum sativum (pea), Oryza sativa (rice), and Gladiolus spp. (Binet et al. 1991; Christensen et al. 1992; Garbarino et al. 1995; Genschik et al. 1994; Joung and Kamo 2006; Kawalleck et al. 1993; Norris et al. 1993; Wang et al. 2000; Xia and Mahon 1998). It has been reported that the well-con-

Corresponding author: Makoto Hayashi; Telephone: +49-89-2180-6375; Fax: +49-89-2180-63858; E-mail: hayashi@1mu.de served intron located in the $5^{\prime}$ untranslated region (UTR) contributes to the high transcriptional activity in A. thaliana, potato, tobacco, and gladiolus (Garbarino et al. 1995; Joung and Kamo 2006; Norris et al. 1993; Plesse et al. 2001). In this study, we attempted to isolate the $L$. japonicus polyubiquitin promoter, whose activity was expected to be high and ubiquitous in L. japonicus, and we constructed the polyubiquitin promoter-based binary vectors.

\section{RESULTS AND DISCUSSION}

Isolation and gene structure of Ljubq1.

To identify a polyubiquitin gene, which potentially possesses the strongest transcriptional activity, we used datasets from The Gene Index Project of L. japonicus at the Computational Biology and Functional Genomics Laboratory of Dana-Farber Cancer Institute and cDNA Array Expression Profiles of $L$. japonicus (Kouchi et al. 2004). In the comparison of Arabidopsis polyubiquitin (UBQ3; AAO00780) to The Gene Index Project of $L$. japonicus, we found 13 tentative consensus sequences (TCs) encoding putative polyubiquitin. Among them, TC14054 consists of 235 expression sequence tags (ESTs) obtained from various tissues, including roots, nodulating roots, immature nodules, mature nodules, flower buds, flowers, pods, and young plants, whereas the others consist of 3 to 47 ESTs. This indicates that TC14054 is a highly and ubiquitously expressed polyubiquitin gene. The cDNA Array Expression Profiles supported this prediction. A polyubiquitin-encoding EST (AV422262), which is expressed the highest in roots, nodulating roots, nodules, and shoots, also belongs to TC14054. Consequently, TC14054 was termed as Ljubql.

To obtain the genomic structure of Ljubql, the sequence of the L. japonicus genomic clone LjT06D07, which included the sequences of $L j u b q 1$, was analyzed. The structure of $L j u b q 1$ is shown in Figure 1A. The Ljubql has two exons, separated by a $5^{\prime}$ UTR intron of $540 \mathrm{bp}$. The length of noncoding exon 1 is predicted to be $47 \mathrm{bp}$ based on $5^{\prime}$ end sequences of an EST (CB828077). A putative TATA box (TATAA) was found at the position of -39 to $-43 \mathrm{bp}$ from the putative transcription initiation site. The exon 2 has an open reading frame of ubiquitin pentamer. Each ubiquitin monomer consists of 76 amino acids and shows $100 \%$ amino acid identity to every other monomer except for the fifth ubiquitin monomer, which has an additional phenylalanine residue at the C-terminal end. An intron at the $5^{\prime}$ UTR is located directly before the translation initiation codon of Ljubql. The nucleotide sequences CAAG/gta at the 
$5^{\prime}$ splice site and cag/ATG at the $3^{\prime}$ splice site were predicted. This structure was similar to the others as descried previously (Binet et al. 1991; Christensen et al. 1992; Garbarino et al. 1995; Genschik et al. 1994; Joung and Kamo 2006; Kawalleck et al. 1993; Norris et al. 1993; Wang et al. 2000; Xia and Mahon 1998).

The promoter activity in roots and nodules of hairy roots.

The promoter activities of four DNA fragments of Ljubq1 were compared in hairy roots. The hairy root transformation is a rapid procedure to generate transgenic roots in $L$. japonicus (Hansen et al. 1989). The 535- or 1,118-bp fragments of Ljubq1 promoter with or without the 5' UTR intron were fused to the $\beta$-glucuronidase (GUS) gene by replacement of the CaMV35S promoter of pBI121 (Jefferson et al. 1987). These four vectors were termed pUB500, pUB1100, pUB500i, and pUB1100i (Fig. 1B).

The hairy roots transformed with four vectors of Ljubq1 showed intense GUS staining in roots and inside of nodules (Fig. 2A to D), whereas the hairy root transformed with pBI121 showed GUS staining in roots and less staining inside of nodules (Fig. 2E). The averages of GUS activity were at almost the same level among four vectors of $L j u b q 1$ and approximately threefold higher than CaMV35S promoter in roots and nodules (Fig. 2G). Notably, the maximum activities of pUB500i and pUB1100i were higher than pUB500 and pUB1100, respectively (Fig. 2G), indicating that the $5^{\prime}$ UTR intron potentially enhanced the transcriptional activities. Taken together, we concluded that the 535-bp fragment of Ljubql with the 5' UTR intron was suffi- cient for a high-level expression of Ljubql, even if the variability of GUS activity (Fig. 2G) and staining patterns (data not shown) for all constructs are taken in account.

The promoter activity in leaves, stems, roots, nodules, and pollen of stable transgenic plants.

To investigate the activities of the Ljubql promoter at ground tissues, stable transgenic plants were generated. As $\mathrm{T}_{0}$ generation, 58 and 73 lines were transformed with pUB500i and pBI121, respectively, and resulting in strong GUS activity in roots or leaves in 18 and 20 lines, respectively. Seed of 13 lines each were obtained from lines of pUB500i and pBI121 transgenic plants in the $\mathrm{T}_{1}$ generation. We used the well-growing $\mathrm{T}_{1}$ lines for the following GUS assay.

The GUS activities of pUB500i-transformed plants were higher than pBI121-transformed plants in all surveyed tissues (Fig. 3). Intense GUS staining was detected inside nodules of pUB500i-transformed plants (Fig. 3G) similar to that observed in the hairy-root experiments. The mature pollen of pUB500itransformed plants showed strong GUS staining (Fig. 3M), whereas those of pBI121-transformed plants showed no GUS staining (Fig. 3N). The weakness of CaMV35S promoter in pollen has been reported in stable transgenic plants of $N$. tabacum (tobacco), Petunia hybrida (petunia), and Lycopersicon esculentum (tomato) (Guerrero et al. 1990; Mascarenhas and Hamilton 1992; van der Leede-Plegt et al. 1992). This result indicates that CaMV35S promoter is unsuitable for ectopic gene expression studies in Lotus japonicus pollen. The averages of GUS activity in leaves, stems, roots, and nodules of pUB500i-transformed
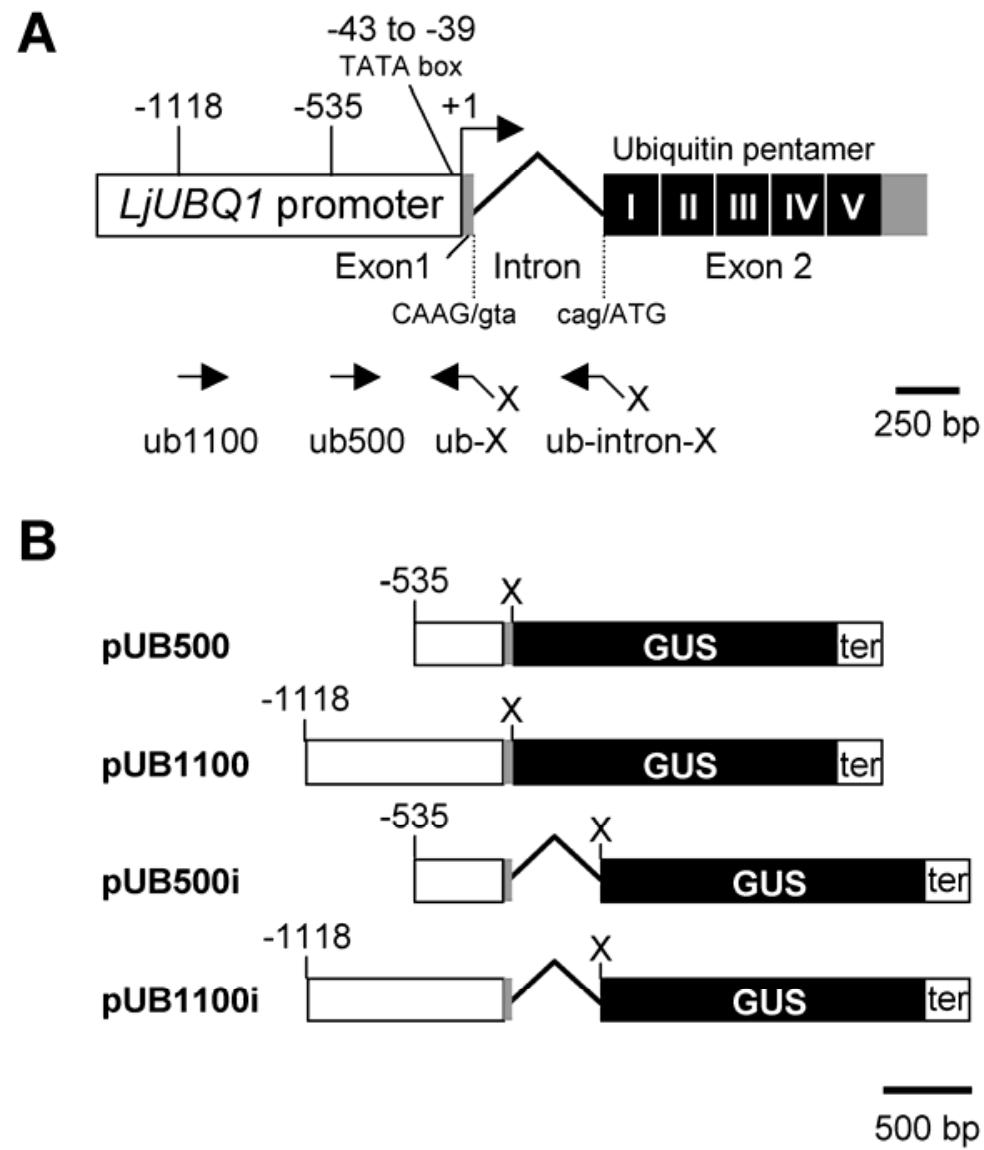

Fig. 1. $L j u b q 1$ structure and the binary vectors for $\beta$-glucuronidase (GUS) assays. A, Gene structure of $L j u b q 1$. $L j u b q 1$ contains two exons separated by a $5^{\prime}$ untranslated region (UTR) intron of $540 \mathrm{bp}$. The noncoding exon 1 is predicted to be $47 \mathrm{bp}$. Exon 2 has an open reading frame of ubiquitin pentamer. The 5 and 3' UTRs are shown as gray box. A putative transcription initiation site is represented as +1 with an arrow. A putative TATA box (TATAA) is located at the position of -39 to $-43 \mathrm{bp}$. The positions of primers to construct the binary vectors are presented below. B, Binary vectors for GUS assays. Additional bases were inserted in the end of Ljubql fragments due to the $\mathrm{XbaI}(\mathrm{X})$ site of the primers; ter = nos terminator. 
plants were approximately eight-, seven-, four-, and fivefold higher, respectively, than those of pBI121-transformed plants (Fig. 3P). These results indicate that the Ljubql promoter is suitable not only for high-level expression in nodules but also for aboveground tissues of L. japonicus.

\section{Ljubq1 promoter-based binary vectors} for overexpression and gene silencing.

pCAMBIA1300 (Cambia, Canberra, Australia) was used as a backbone in the general structure of Ljubql promoter-based binary vectors. pCAMBIA1300 is a derivative of $p P Z P$. The features of $p P Z P$ are high copy replication in Escherichia coli and high stability in Agrobacterium spp. without antibiotics (Hajdukiewicz et al. 1994). For bacterial selection (in E. coli or Agrobacterium spp.), kanamycin is available. The plantselectable marker gene ( $h p t I I$ for hygromycin resistant) is located next to the left border. Because the T-DNA transfer is initiated from the right border, the hygromycin resistance means that an entire T-DNA is transferred into plants. The hygromycin resistance gene is more suitable for transformation
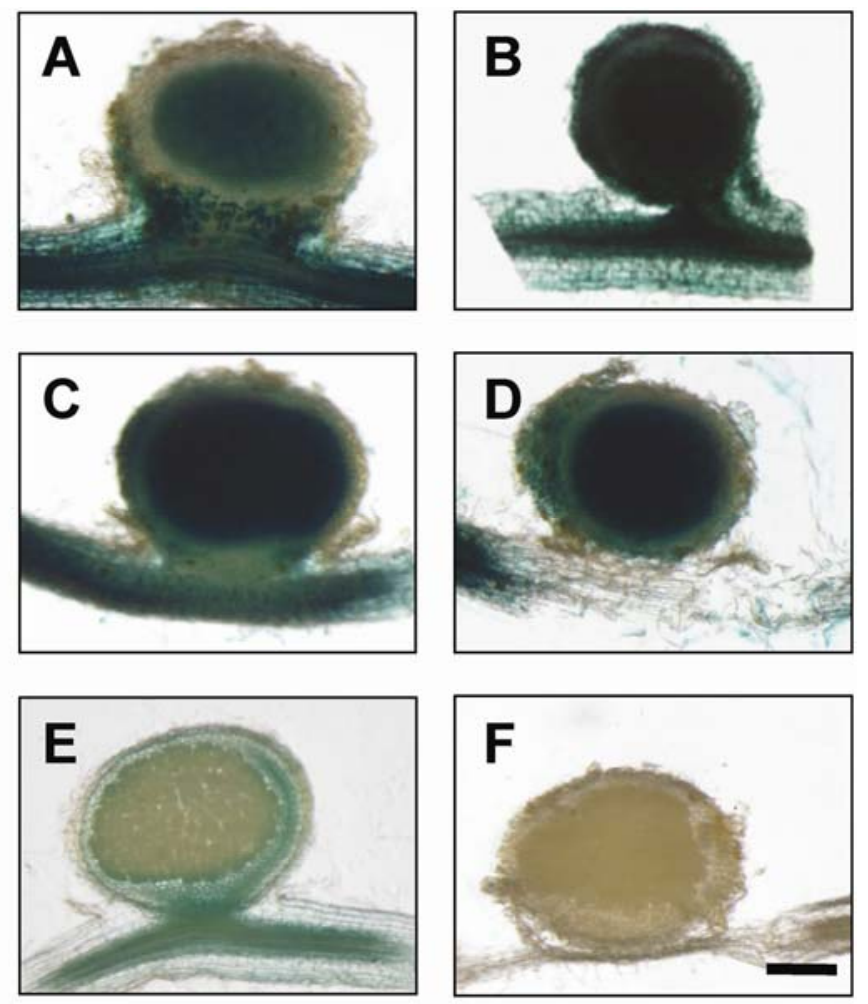

\section{G}

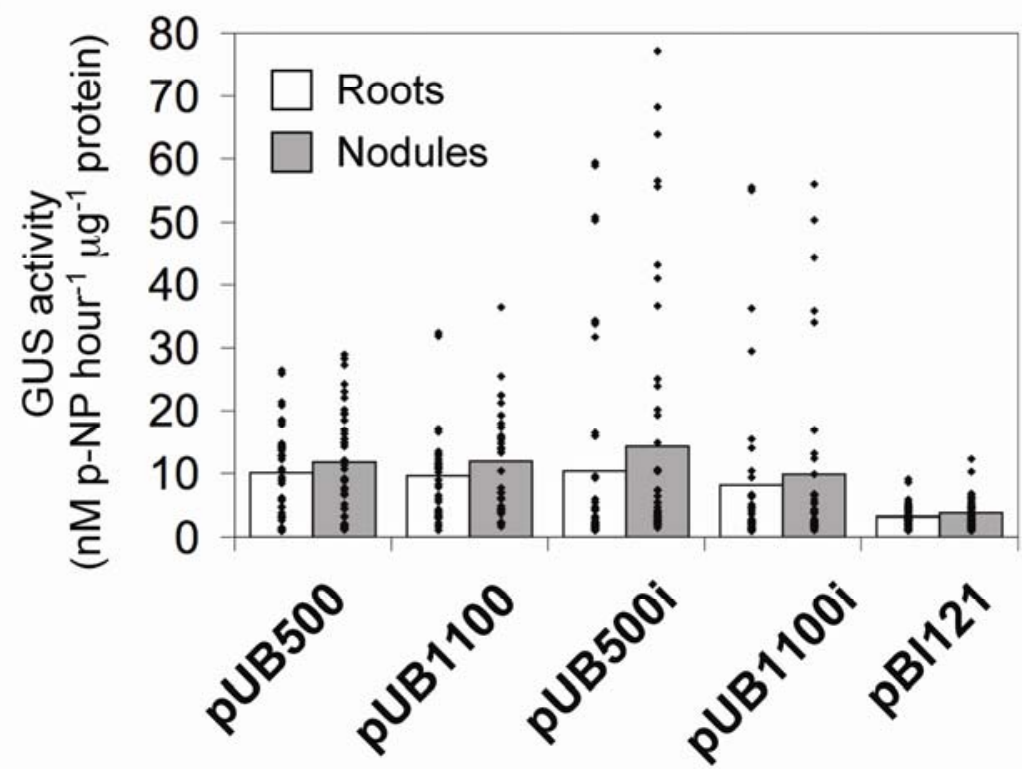

Fig. 2. Promoter activity in hairy roots. A through F, Pictures of $\beta$-glucuronidase (GUS)-stained nodule sections. Nodules were obtained from the plants transformed with A, pUB500; B, pUB1100; C, pUB500i; D, pUB1100i; and E, pBI121; and F, untransformed MG-20. G, GUS activity in roots and nodules. The white and gray bars represent the average GUS activity in roots and nodules, respectively $(n=60)$. Black dots represent individual values of GUS activity from independent roots. There are significant differences between pUB500i and pBI121 both for roots and nodules $(P<0.01)$. Bar $=250 \mu \mathrm{m}$. 


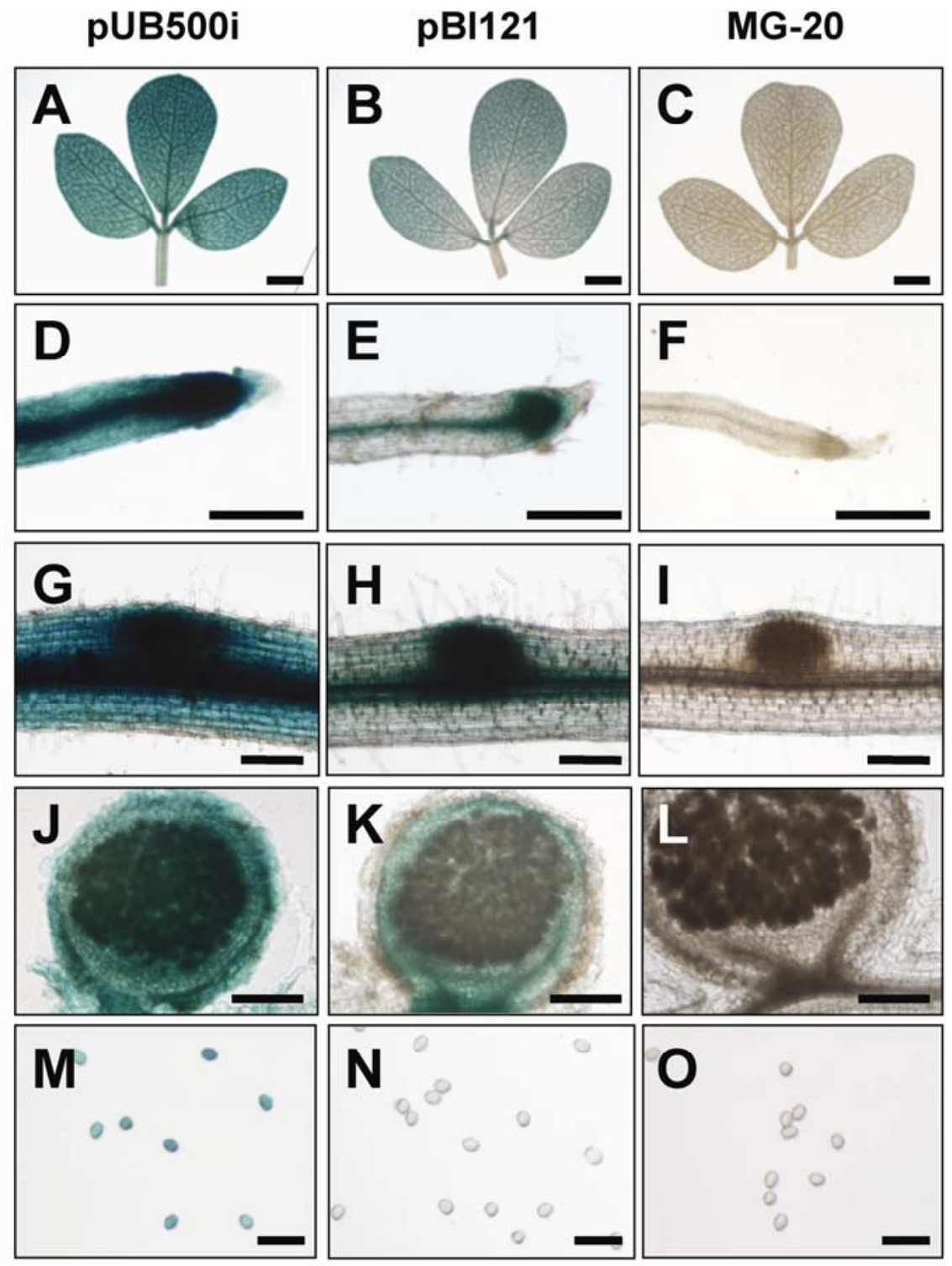

$\mathbf{P}$

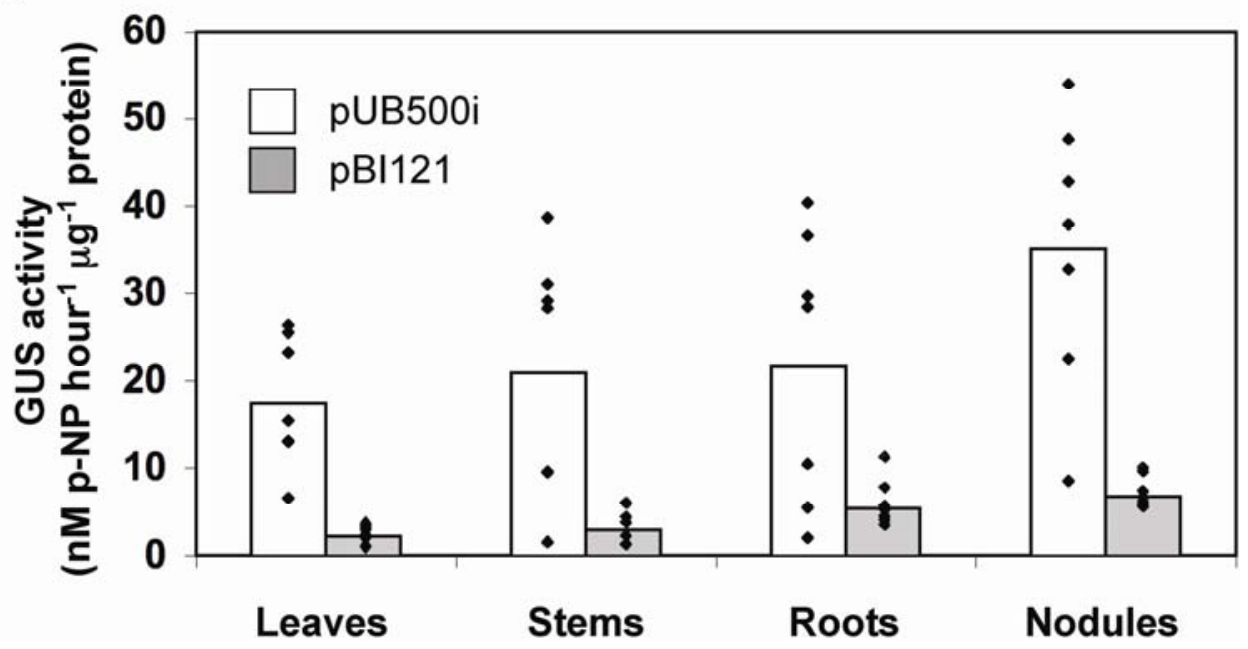

Fig. 3. Promoter activity in the stable transgenic plants. Pictures of $\beta$-glucuronidase (GUS)-stained A through $\mathbf{C}$, leaves; $\mathbf{D}$ through $\mathbf{F}$, root tips; $\mathbf{G}$-I, nodule primordia; J through $\mathbf{L}$, nodule sections; and $\mathbf{M}$ through $\mathbf{O}$, mature pollen obtained from the stable transgenic plants ( $\mathrm{T}_{1}$ ) with $\mathbf{A}$, D, G, J, and $\mathbf{M}$, pUB500i; $\mathbf{B}, \mathbf{E}, \mathbf{H}, \mathbf{K}$, and N, pBI121; and C, F, I, L, and $\mathbf{O}$, untransformed MG-20. P, GUS activity in leaves, stems, roots, and nodules. The white and gray bars represent the average GUS activity of each tissue transformed with pUB500i and pBI121, respectively $(n=7)$. Black dots represent individual value of GUS activity from independent transgenic lines. There are significant differences between pUB500i and pBI121 for all combinations $(P<0.01$; except for roots, $P$ $<0.02$ ) . Bars = A through $\mathbf{F}, 1 \mathrm{~mm} ; \mathbf{G}$ through $\mathbf{L}, 250 \mu \mathrm{m}$; and $\mathbf{M}$ through $\mathbf{O}, 50 \mu \mathrm{m}$. 
of L. japonicus than the kanamycin resistance gene because the hygromycin selection results in less escape than the kanamycin selection (Handberg and Stougaard 1992). Therefore, the hygromycin resistance gene was selected.

We developed four binary vectors supported with GATEWAY conversion technology. For overexpression of a transgene under the Ljubql promoter, two binary destination vectors are available (Fig. 4). pUB-GW-GFP and pUB-GW-Hyg have the GATEWAY recombination site between the Ljubql promoter plus the 5' UTR intron and the nos terminator (Fig. 4). For gene silencing under the Ljubql promoter, two binary destination vectors were constructed (Fig. 4): pUB-GWS-GFP and pUB-GWS-Hyg have tandem GATEWAY cassettes, in opposite orientation to each other. The intron 1 of AtWRKY33 is located between the cassettes. These vectors are able to produce double-stranded RNA with the intron, whose structure leads to efficient gene silencing (Wesley et al. 2001). We also constructed pUB-GFP and pUB-Hyg for conventional cloning strategies without GATEWAY technology. The vectors shown in Figure 4 were deposited at the National BioResource Project Legume Base and are available upon request. The sequence of the genome clone LjT06D07 was deposited at the DNA Data Bank of Japan (DDBJ) under accession numbers
AP009382 and AP009383. The full sequences of pUB-GWGFP, pUB-GW-Hyg, pUB-GWS-GFP, pUB-GWS-Hyg, pUBGFP, and pUB-Hyg were deposited at the DDBJ under accession numbers AB303064, AB303065, AB303066, AB303067, AB303068, and AB303069, respectively.

As a test, the gus reporter gene was cloned into pUB-GWGFP and transformed into plants. The expected GUS staining was observed in nodules of transformed hairy roots (Fig. 5A). To test the efficiency of gene silencing, a partial sequence of the gus gene (approximately $450 \mathrm{bp}$ ) was cloned into pUBGWS-GFP and the construct was transformed into the stably gus-transformed plants. Hairy root transformation often produces chimeric transgenic roots; therefore, we classified the transgenic roots with the pattern of GFP intensity, which is a transformation marker. In entirely strong GFP-expressed roots and in chimeric or weak GFP-expressed roots, 84 and $65 \%$ of roots were silenced, respectively (Table 1; Fig. 5B). This result suggests that the RNAi vectors are useful for gene silencing. In the case of no GFP expressed roots, $14 \%$ of roots were silenced (Table 1). This result may be due to the fact that the RNAi construct is transformed; however, the construct for GFP fails to be transformed in a certain frequency. Interestingly, we observed the GUS staining at $15 \%$ of the root tips even though

\section{pUB-GW-GFP}

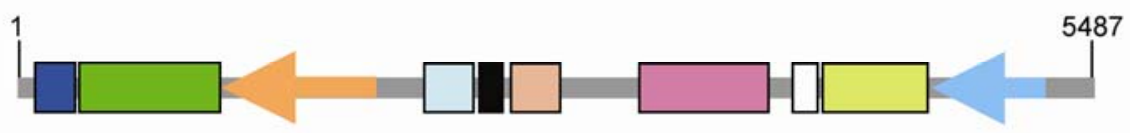

pUB-GW-Hyg

\section{$500 \mathrm{bp}$}

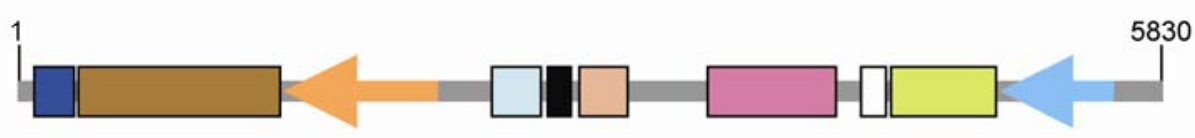

pUB-GWS-GFP

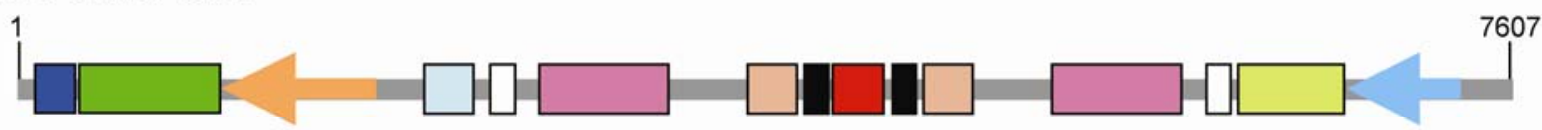

\section{pUB-GWS-Hyg}

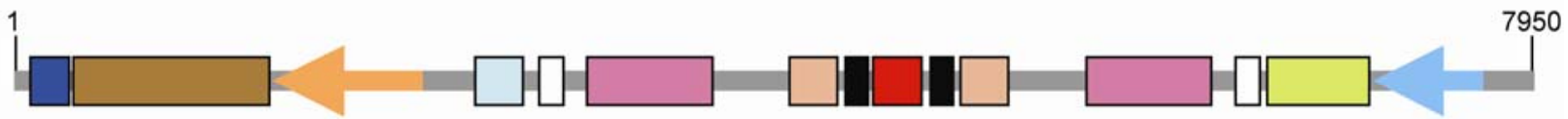

\section{pUB-GFP}

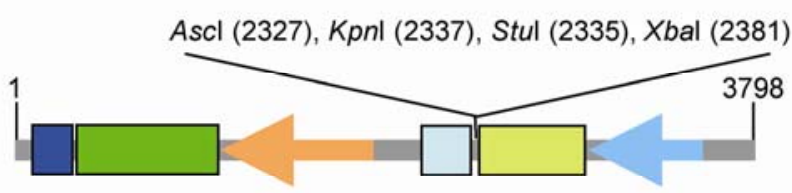

pUB-Hyg
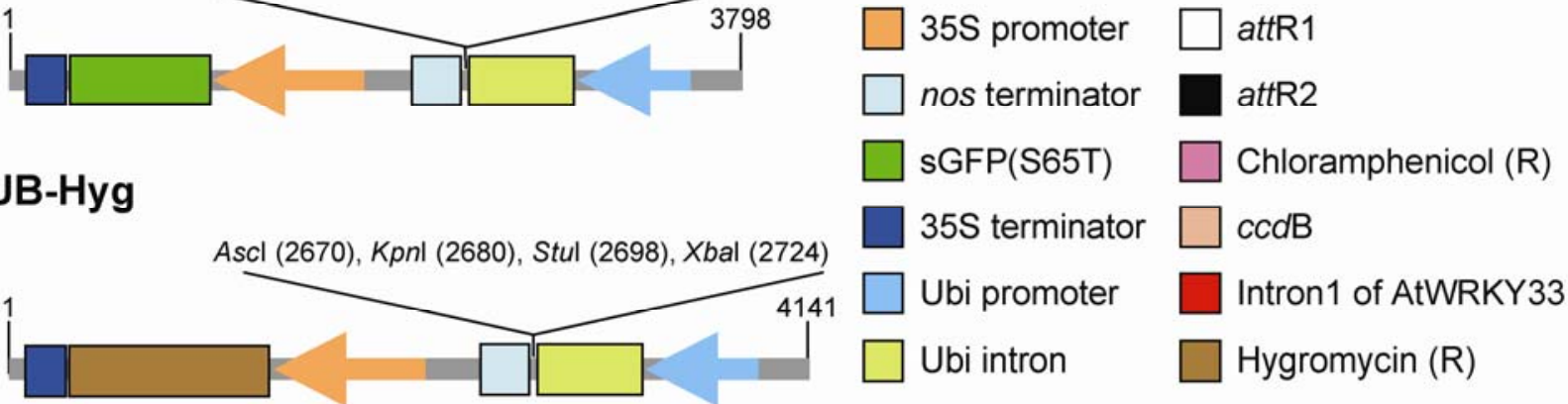

Fig. 4. Maps of Ljubq1 promoter-based binary vectors. The binary vectors are represented as linear segments from the left to right borders. These constructs were based on a vector of which the 535-bp fragment of Ljubq1 with the 5' untranslated region intron that was cloned into pCAMBIA1300. The hygromycin resistance gene was originated from the backbone vector, pCAMBIA1300. 
the other parts of the plants were silenced (Fig. 5C), indicating that root meristem is less susceptible to gene silencing. Similarly, gene silencing was not accomplished in nodule primordia, although other tissues were silenced (Fig. 5D). The mature nodules were silenced efficiently (Fig. 5E and F), except for a reminiscent GUS staining in nodule vasculatures (Fig. 5E, arrowhead).

The Ljubql promoter-based binary vectors are now available for overexpression and gene silencing. The newly developed binary vectors are compatible with GATEWAY conversion technology that allows high-throughput cloning of interesting genes or DNA sequences. These vectors will help gene function analysis of L. japonicus.

\section{MATERIALS AND METHODS}

\section{Transformation of $\mathbf{L}$. japonicus.}

L. japonicus accession Miyakojima MG-20 was used in this study. Hairy roots were induced using Agrobacterium rhizogenes LBA1334 (Offringa et al. 1986) or AR1193 (Stougaard et al. 1987) as described previously (Díaz et al. 2005). Stable transgenic plants were generated using A. tumefaciens LBA4404 (Jen and Chilton 1986) as described previously (Kato et al. 2005). Binary vectors were electroporated into bacteria using BTX Electro Cell Manipulator 600 (BTX Inc., San Diego, CA, U.S.A.) according to the manufacturer's instructions.

Transgenic plants were grown with nutrients premixed in Power Soil (Kureha Chemical Co., Tokyo) in a cultivation chamber (LPH-350S; Nippon Medical \& Chemical Instruments Co., Ltd., Osaka, Japan; and MLR-351H; Sanyo Electric Co., Ltd., Osaka, Japan) under a day-and-night cycle of 16 and $8 \mathrm{~h}$ and temperatures of 26 and $23^{\circ} \mathrm{C}$, respectively.

\section{Rhizobial inoculation.}

Surface-sterilized seed were germinated on $1 \%$ agar plate. The plates were covered with aluminum foil and set vertically for 2 days. The aluminum foil was removed and subsequently incubated for two more days under light in the cultivation chamber. The 4-day-old seedlings were transplanted to B\&D nitrogen-free medium (Broughton and Dilworth 1971) with 1\% Bacto agar (Becton Dickinson, Rutherford, NJ, U.S.A.) plate. After 3 days of incubation, Mesorhizobium loti MAFF303099 was inoculated. After 3 weeks of incubation, the leaves, stems, roots, and nodules were collected and used for GUS assays.

Plants with hairy roots were transplanted to B\&D nitrogenfree medium with $1 \%$ bacto agar. After 3 days of incubation, M. loti MAFF303099 was inoculated. After 3 weeks of incubation, the roots and nodules were collected and used for GUS assays.

\section{GUS assays.}

Histochemical GUS staining was performed as described previously (Stomp 1992) with minor modifications for leaf staining. Leaves were incubated in $90 \%$ acetone for $15 \mathrm{~min}$ at $-20^{\circ} \mathrm{C}$ before staining. After $4 \mathrm{~h}$ of staining at $37^{\circ} \mathrm{C}$, the samples were washed twice with $100 \mathrm{mM} \mathrm{NaPO}_{4}(\mathrm{pH}$ 7.0). The leaves were bleached with $70 \%$ ethanol at room temperature. Nodules were embedded with $6 \%$ agarose and the sections were prepared with a vibrating blade microtome (VT1000S; Leica Microsystems, Wetzlar, Germany). Mature pollen released from the anthers was stained for $2 \mathrm{~h}$. The samples were observed with a light microscope (BX-50; Olympus, Tokyo) and photographed by a digital camera (DP70; Olympus). The activity of GUS in $T_{1}$ plants was measured with $p$-nitrophenol as described previously (Jefferson et al. 1986).

Table 1. Efficiency of gene silencing of $\beta$-glucuronidase (gus) by pUBGWS-GFP

\begin{tabular}{lc}
\hline GFP intensity in hairy roots & $\begin{array}{c}\text { Efficiency (\%) } \\
\text { (no. of roots unstained/total) }\end{array}$ \\
\hline Entirely strong & $84(16 / 19)$ \\
Chimeric or weak & $65(11 / 17)$ \\
None & $14(4 / 28)$ \\
\hline
\end{tabular}

${ }^{\mathrm{a}} \mathrm{GFP}=$ green fluorescent protein. Hairy roots were stained for $4 \mathrm{~h}$ and unstained roots were counted as silenced roots.
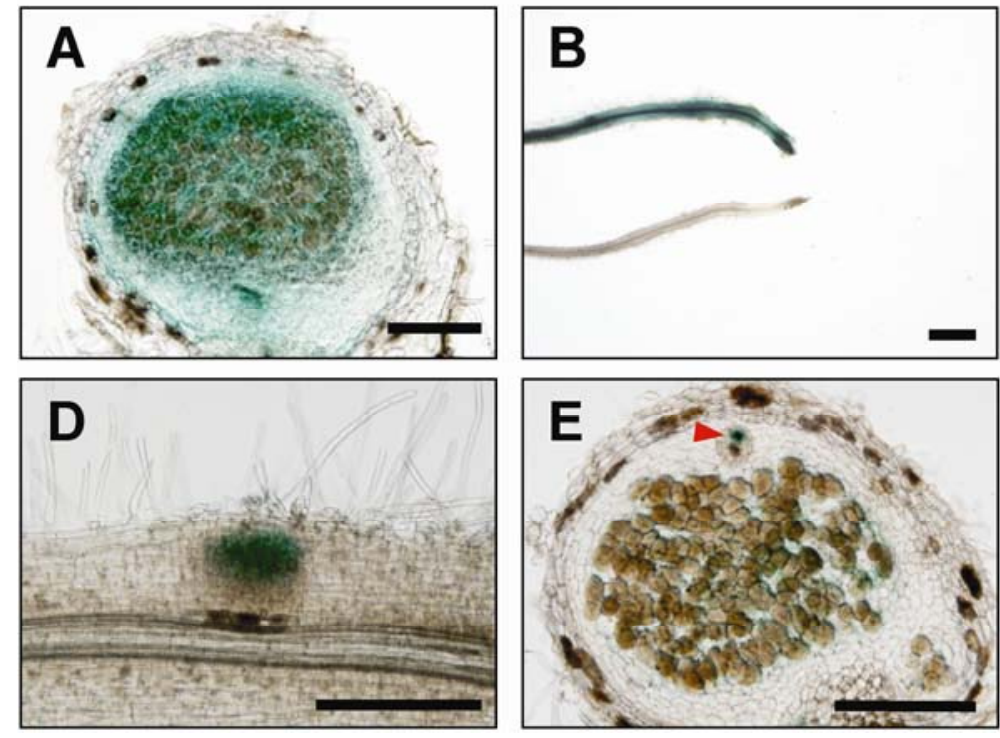
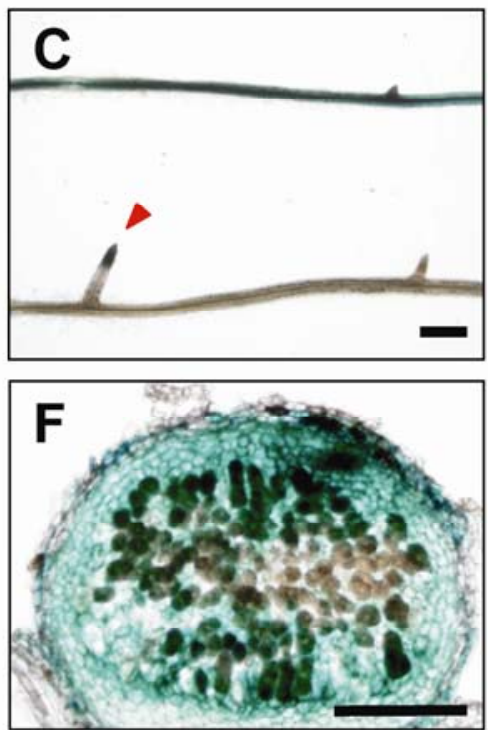

Fig. 5. Overexpression and gene silencing of $\beta$-glucuronidase (gus) transformed with Ljubq1 promoter-based binary vectors in hairy roots. A, Overexpression of gus with pUB-GW-GFP. The gus of pBI121 was cloned into pUB-GW-GFP and transformed. B through F, Gene silencing of gus with pUB-GWS-GFP. Stably gus-transformed plants generated with pUB500i were used for the hairy root transformation. B and C, GFP-negative roots (upper) or positive roots (lower) were stained for $4 \mathrm{~h}$. The pair of roots in each picture originated from the same shoot. C, Nonsilenced root tip was indicated by an arrowhead. D, A nodule primordium from the GFP-positive root. Mature nodules from the E, GFP-positive and $\mathbf{F}$, GFP-negative roots. An arrowhead in E indicates a vascular bundle. Bars $=\mathbf{A}, \mathbf{D}$ through $\mathbf{F}, 250 \mu \mathrm{m}$ and $\mathbf{B}$ and $\mathbf{C}, 1 \mathrm{~cm}$. 
In silico analysis.

The Gene Index Project of L. japonicus and cDNA Array Expression Profiles of L. japonicus (Kouchi et al. 2004) were used to identify the polyubiquitin gene, which potentially possessed the strongest transcriptional activity.

\section{Construction of binary vectors.}

PCR was performed with KOD-Plus-DNA polymerase (Toyobo, Osaka, Japan) according to the manufacturer's instructions. The polymerase chain reaction (PCR) products or digested samples with restriction enzymes were purified with QIAquick PCR purification kit (Qiagen, Hilden, Germany) according to the manufacturer's instructions. DNA ligation kit (version 2.1; Takara, Kyoto, Japan) was used for DNA ligation according to the manufacturer's instructions. For the construction of pUB500, pUB1100, pUB500i, and pUB1100i, the CaMV35S promoter of pBI121 (Jefferson et al. 1987) was replaced by fragments of L. japonicus ubiquitin (Ljubq1) as follows. First, the regions of the Ljubql promoter and the intron were amplified from a L. japonicus genomic clone, LjT06D07, with the primers below: ub500, 5'-GGAGAGAGGATTTTGAGGAA-3' and ub-X, 5'-CTAGTCTAGAGAATTGTTAGGGTTGGGAAC3' for pUB500; ub1100, 5'-CTTCCATACTTTCATGGTGTG-3' and $\mathrm{ub}$-X for pUB1100; ub500 and ub-intron-X, 5'-CTAGTCT AGACTGTAATCACATCAACAACAGA-3' for pUB500i; and ub1100 and ub-intron-X for pUB1100i (Fig. 1). The fragments were digested with XbaI. pBI121 was digested with HindIII and treated with Klenow fragment (Takara) to create blunt ends. Then the treated pBI121 was digested with $X b a I$ and ligated into each fragment of Ljubql.

To construct binary destination vectors supported with GATEWAY conversion technology (Invitrogen, Carlsbad, CA, U.S.A.) as shown in Figure 4, pCAMBIA1300 (Cambia) was used as a backbone in the general structure of Ljubql promoter-based binary vectors. As a selectable marker for plant transformation, we used the hygromycin-selectable marker gene of pCAMBIA1300 or sGFP (S65T) gene of modified pHKN29,pHKN29r (Kumagai and Kouchi 2003; Niwa et al. 1999). As by-products, we also constructed pUB-GFP and pUB-Hyg for conventional cloning strategies without GATEWAY technology.

The open reading frame of the gus gene on pBI121 was amplified with GUScds-attB1, AAAAAGCAGGCTATGTTAC GTCCTGTAGAAAC and GUScds-attB2, AGAAAGCTGGGT TCATTGTTTGCCTCCCTGCT (the underlines indicate specific sequences of the gus gene). The PCR products were reamplified with attB1 adapter, 5'-GGGGACAAGTTTGTACAAA AAAGCAGGCT-3' and $a t t$ B2 adapter, 5'-GGGGACCACTTT GTACAAGAAAGCTGGGT-3'. The attB recombination sites flanking the PCR product were subcloned into $\mathrm{pDONR} / \mathrm{Zeo}$ (Invitrogen) using BP clonase II enzyme mix (Invitrogen) according to the manufacturer's instructions. The gus sequence on the pDONR/Zeo subsequently was transferred into pUBGW-GFP using LR clonase II enzyme mix (Invitrogen) according to the manufacturer's instructions.

The partial sequence of the gus gene on pBI121 (445 bp) was amplified with GUS-attB1, 5'-AAAAAGCAGGCTGGGC CAACAGTTCCTGATTA- ${ }^{\prime}$ ' and GUS-attB2, 5'-AGAAAGCT GGGTCGAAATATTCCCGTGCACTT-3' (the underlines indicate specific sequences of the gus gene). The PCR product was reamplified with attB1 adapter and attB2 adapter. The partial sequence of the gus gene was transferred into pUB-GWS-GFP.

\section{ACKNOWLEDGMENTS}

We thank L. Rose for critical reading of the manuscript, H. Seki and T. Muranaka for providing the GATEWAY-compatible RNAi cassette, and H. Kumagai and H. Kouchi for GFP version of pCAMBIA1300. This work was supported by the CREST Program from the Japan Science and Technology Agency to T. Maekawa and M. Hayashi.

\section{LITERATURE CITED}

Auriac, M.-C., and Timmers, A. C. J. 2007. Nodulation studies in the model legume Medicago truncatula: Advantages of using the constitutive EF1 $\alpha$ promoter and limitations in detecting fluorescent reporter proteins in nodule tissues. Mol. Plant-Microbe Interact. 20:1040-1047.

Binet, M. N., Weil, J. H., and Tessier, L. H. 1991. Structure and expression of sunflower ubiquitin genes. Plant Mol. Biol. 17:395-407.

Broughton, W. J., and Dilworth, M. J. 1971. Control of leghaemoglobin synthesis in snake beans. Biochem. J. 125:1075-1080.

Burke, T. J., Callis, J., and Vierstra, R. D. 1988. Characterization of a polyubiquitin gene from Arabidopsis thaliana. Mol. Gen. Genet. 213:435-443.

Christensen, A. H., Sharrock, R. A., and Quail, P. H. 1992. Maize polyubiquitin genes: Structure, thermal perturbation of expression and transcript splicing, and promoter activity following transfer to protoplasts by electroporation Plant Mol. Biol. 18:675-689.

Díaz, C. L., Grønlund, M., Schulaman, H. R. M., and Spaink, H. P. 2005 Induction of hairy roots for symbiotic gene expression studies. Pages 261-277 in: Lotus japonicus Handbook. A. J. Márguez, ed. Springer, Dordrecht, The Netherlands.

Garbarino, J. E., Oosumi, T., and Belknap, W. R. 1995. Isolation of a polyubiquitin promoter and its expression in transgenic potato plants. Plant Physiol. 109:1371-1378.

Genschik, P., Marbach, J., Uze, M., Feuerman, M., Plesse, B., and Fleck, J. 1994. Structure and promoter activity of a stress and developmentally regulated polyubiquitin-encoding gene of Nicotiana tabacum. Gene 148:195-202.

Guerrero, F. D., Crossland, L., Smutzer, G. S., Hamilton, D. A., and Mascarenhas, J. P. 1990. Promoter sequences from a maize pollen-specific gene direct tissue-specific transcription in tobacco. Mol. Gen. Genet. 224:161-168.

Hajdukiewicz, P., Svab, Z., and Maliga, P. 1994. The small, versatile $p P Z P$ family of Agrobacterium binary vectors for plant transformation. Plant Mol. Biol. 25:989-994

Handberg, K., and Stougaard, J. 1992. Lotus japonicus, an autogamous, diploid legume species for classical and molecular genetics. Plant J. 2:487-496.

Hansen, J., Jorgensen, J. E., Stougaard, J., and Marcker, K. A. 1989. Hairy roots - a short cut to transgenic root nodules. Plant Cell Rep. 8:12-15.

Jefferson, R. A., Burgess, S. M., and Hirsh, D. 1986. $\beta$-Glucuronidase from Escherichia coli as a gene-fusion marker. Proc. Natl. Acad. Sci. U.S.A. 83:8447-8451.

Jefferson, R. A., Kavanagh, T. A., and Bevan, M. W. 1987. GUS fusions: Beta-glucuronidase as a sensitive and versatile gene fusion marker in higher plants. EMBO (Eur. Mol. Bio. Organ.) J. 6:3901-3907.

Jen, G. C., and Chilton, M. D. 1986. Activity of T-DNA borders in plant cell transformation by mini-T plasmids. J. Bacteriol. 166:491-499.

Joung, Y. H., and Kamo, K. 2006. Expression of a polyubiquitin promoter isolated from Gladiolus. Plant Cell Rep. 25:1081-1088.

Kato, T., Goto, Y., Ono, K., Hayashi, M., and Murooka, Y. 2005. Expression of a major house dust mite allergen gene from Dermatophagoides farinae in Lotus japonicus accession Miyakojima MG-20. J. Biosci. Bioeng. 99:165-168.

Kawalleck, P., Somssich, I. E., Feldbrugge, M., Hahlbrock, K., and Weisshaar, B. 1993. Polyubiquitin gene expression and structural properties of the ubi4-2 gene in Petroselinum crispum. Plant Mol. Biol. 21:673-684.

Kouchi, H., Shimomura, K., Hata, S., Hirota, A., Wu, G.-J., Kumagai, H., Tajima, S., Suganuma, N., Suzuki, A., Aoki, T., Hayashi, M., Yokoyama, T., Ohyama, T., Asamizu, E., Kuwata, C., Shibata, D., and Tabata, S. 2004. Large-scale analysis of gene expression profiles during early stages of root nodule formation in a model legume, Lotus japonicus. DNA Res. 11:263-274.

Kumagai, H., and Kouchi, H. 2003. Gene silencing by expression of hairpin RNA in Lotus japonicus roots and root nodules. Mol. Plant-Microbe Interact. 16:663-668

Mascarenhas, J. P., and Hamilton, D. A. 1992. Artifacts in the localization of GUS activity in anthers of petunia transformed with a CaMV 35SGUS construct. Plant J. 2:405-408.

Niwa, Y., Hirano, T., Yoshimoto, K., Shimizu, M., and Kobayashi, H. 1999. Non-invasive quantitative detection and applications of non-toxic, S65Ttype green fluorescent protein in living plants. Plant J. 18:455-463.

Norris, S. R., Meyer, S. E., and Callis, J. 1993. The intron of Arabidopsis thaliana polyubiquitin genes is conserved in location and is a quantitative determinant of chimeric gene expression. Plant Mol. Biol. 21:895-906. 
Obertello, M, Santi, C, Sy, M.-O., Laplaze, L., Auguy, F., Bogusz, D., and Franche, C. 2005 Comparison of four constitutive promoters for the expression of transgenes in the tropical nitrogen-fixing tree Allocasuarina verticillata. Plant Cell Rep. 24:540-548.

Offringa, I. A., Melchers, L. S., Regensburg-Tuink, A. J., Costantino, P., Schilperoort, R. A., and Hooykaas, P. J. 1986. Complementation of Agrobacterium tumefaciens tumor-inducing aux mutants by genes from the $\mathrm{T}_{\mathrm{R}}$-region of the Ri plasmid of Agrobacterium rhizogenes. Proc. Natl. Acad. Sci. U.S.A. 83:6935-6939.

Plesse, B., Criqui, M. C., Durr, A., Parmentier, Y., Fleck, J., and Genchik, P. 2001. Effects of the polyubiquitin gene Ubi.U4 leader intron and first ubiquitin monomer on reporter gene expression in Nicotiana tabacum. Plant Mol. Biol. 45:655-667.

Quandt, H.-J., Pühler, A., and Broer, I. 1993. Transgenic root nodules of Vicia hirsuta: A fast and efficient system for the study of gene expression in indeterminate-type nodules. Mol. Plant-Microbe Interact. 6:699-706.

Samac, D. A., Tesfaye, M., Dornbusch, M., Saruul, P., and Temple, S. J. 2004 A comparison of constitutive promoters for expression of transgenes in alfalfa (Medicago sativa). Transgenic Res. 13:349-361.

Smalle, J., and Vierstra, R. D. 2004. The ubiquitin 26S proteasome proteolytic pathway. Annu. Rev. Plant Biol. 55:555-590.

Stomp, A. M. 1992. Histochemical localization of $\beta$-glucuronidase. Pages 103-113 in: GUS Protocols: Using the GUS Gene as a Reporter of Gene Expression. S. R. Gallagher, ed. Academic Press, San Diego, CA, U.S.A.

Stougaard, J., Abildsten, D., and Marcker, K. A. 1987. The Agrobacterium rhizogenes $\mathrm{pRi}$ TL-DNA segment as a gene vector system for transfor- mation of plants. Mol. Gen. Genet. 207:251-255.

Udvardi, M. K., Tabata, S., Parniske, M., and Stougaard, J. 2005. Lotus japonicus: Legume research in the fast lane. Trends. Plant Sci. 10:222228.

van der Leede-Plegt, L. M., Ven, B .C. E., Bino, R. J., Salm, T. P. M., and Tunen, A. J. 1992. Introduction and differential use of various promoters in pollen grains of Nicotiana glutinosa and Lilium longiflorum. Plant Cell Rep. 11:20-24.

Wang, J. L., Jiang, J. D., and Oard, J. H. 2000. Structure, expression and promoter activity of two polyubiquitin genes from rice (Oryza sativa L.). Plant Sci. 156:201-211.

Wesley, S. V., Helliwell, C. A., Smith, N. A., Wang, M. B., Rouse, D. T., Liu, Q., Gooding, P. S., Singh, S. P., Abbott, D., Stoutjesdijk, P. A., Robinson, S. P., Gleave, A. P., Green, A. G., and Waterhouse, P. M. 2001. Construct design for efficient, effective and high-throughput gene silencing in plants. Plant J. 27:581-590.

Xia, X., and Mahon, J. 1998. Pea polyubiquitin genes: (I) structure and genomic organization. Gene 215:445-452.

\section{AUTHOR-RECOMMENDED INTERNET RESOURCES}

National BioResource Project (NBRP) Legume Base: www.legumebase.agr.miyazaki-u.ac.jp/

The Gene Index Project: compbio.dfci.harvard.edu/tgi/

Kazusa DNA Research Institute cDNA Array Expression Profile website: www.kazusa.or.jp/en/plant/lotus/EST/cDNA.html 